\title{
Klebsiella pneumoniae Bacteremia Presenting on a Neonatal Intensive Care Unit during the First Week of Life
}

\author{
Majid Malaki ${ }^{1}$ \\ ${ }^{1}$ Sevome Shaban Hospital, Tehran, Iran \\ J Pediatr Infect Dis 2015;10:12-15.
}

\begin{abstract}
Address for correspondence Majid Malaki, Assistant Professor of Pediatric, Sevome Shaban Hospital, Tehran, Iran (e-mail: madjidmalaki@gmail.com).
\end{abstract}

\begin{abstract}
Klebsiella pneumoniae is a major cause of neonatal infection that is associated with significant morbidity and mortality. Here, we define clinical aspects and outcomes of Klebsiella infection in our center. All infants newly admitted to our neonatal intensive care unit over a 1-year period were included. A blood culture was collected from a neonate during the first week of life who had any abnormal clinical and/or laboratory findings consistent with infection. Of 805 neonates, 51 (6\%) had Klebsiella bacteremia in the first week of life, mostly before the 5 th day (69\%). Klebsiella bacteremia was 12 times more common in neonates weighing less than $2,500 \mathrm{~g}$ compared with those weighing

Keywords

- complication

- hyperbilirubinemia

- incidence

- Klebsiella pneumoniae

- neonate $2,500 \mathrm{~g}$ or more. Overall, $41 \%$ of patients had subtle clinical findings; hyperbilirubinemia (86\%), elevated C-reactive protein concentration (68\%), and thrombocytopenia (60\%) were the most common abnormalities found on laboratory testing, with all three being abnormal in around 50\% of cases. Meningitis, necrotizing enterocolitis, and septic arthritis were the main complications occurring in $29 \%$ of cases. The overall mortality rate was $25 \%$, and low birth weight was found to be a major risk for mortality.
\end{abstract}

\section{Introduction}

Bacteremia is a common problem in neonatal intensive care units (NICUs) even in developed countries, and is associated with significant morbidity and mortality. ${ }^{1}$ Prematurity and prolonged hospital stay are important predisposing factors for neonatal bacteremia, which can be either early onset or late onset. ${ }^{2-5}$ Klebsiella pneumoniae, a gram-negative bacterium that is a component of the normal gastrointestinal flora of humans, is one important cause of neonatal bacteremia ${ }^{6-8}$; indeed in some centers, it is the most common cause of neonatal sepsis. ${ }^{8-12}$

We reviewed the incidence of, risks factors for, and complications of $K$. pneumoniae bacteremia in our NICU.

received

October 7, 2014

accepted after revision

January 15, 2014

\section{Patients and Methods}

During the 1-year study period, 805 eligible neonates were admitted in NICU on the first day of life and stayed for more than 7 days. Patients who were excluded were those transferred from surgical wards or other hospitals, those admitted for a surgical procedure, those with major congenital malformations, and those whose mothers had prolonged rupture of membrane. A blood culture, collected on admission, and further blood cultures were undertaken thereafter where there were new clinical or laboratory findings suggestive of infection.

Clinical features of neonatal sepsis were as follows: (1) hypothermia or fever; (2) lethargy, poor cry, and refusal to suck; (3) poor perfusion, prolonged capillary refill time,

Copyright $\odot 2015$ by Georg Thieme Verlag KG, Stuttgart · New York
DOI http://dx.doi.org/ 10.1055/s-0035-1554971. ISSN 1305-7707. 
hypotension, or shock; (4) hypotonia and absent neonatal reflexes; (5) bradycardia or tachycardia; (6) respiratory distress, apnea, and gasping respiration; (7) signs of meningitis (bulging anterior fontanelle, blank look, high-pitched cry, excess irritability, not arousal, comatose, seizures, and neck retraction); (8) feed intolerance, vomiting, diarrhea, abdominal distension, hepatomegaly, paralytic ileus, and suspicion of necrotizing enterocolitis (NEC); (9) bleeding, petechiae, or purpura; and (10) skin changes, including pustules, abscesses, sclerema, mottling, umbilical redness, and discharge.

Laboratory features of neonatal sepsis were as follows: hypoglycemia or hyperglycemia; metabolic acidosis; hyperbilirubinemia; acute renal failure; elevated C-reactive protein (CRP) concentration ( $>1.5 \mathrm{mg} / \mathrm{dL})$; leukopenia $(<5,000$ cell/ $\left.\mathrm{mm}^{3}\right)$; and thrombocytopenia $\left(<100,000 \mathrm{cell} / \mathrm{mm}^{3}\right)$. All this information matched in patients with approved blood culture for Klebsiella. Patients' survival followed and fatal cases recorded beside to complications which occurred before leaving hospital.

Blood cultures were collected and processed, and isolates identified, using standard techniques. ${ }^{13}$ Empiric antibiotic treatment was with ampicillin plus either gentamicin or cefotaxime, narrowed to organism-specific drugs as soon as possible.

For the study, clinical data were obtained by retrospective case note review. Nosocomial infection was considered to be present if onset of infection was found after 72 hours of birth. Statistical Package for Social Sciences (SPSS version 15), and Tindependent and chi-square tests; $p$ values of less than 0.05 were considered to be significant.

\section{Results}

Of 805 neonates, 51 (6\% of all admitted cases) had bacteremia with K. pneumonia presenting in the first week of life. Overall, $63 \%$ of those affected were in very low-birth-weight (VLBW) group $(<1,500 \mathrm{~g})$. The incidence of Klebsiella bacteremia in neonate weighing 1,500 to $2,500 \mathrm{~g}$ was $33 \%$ (32/95), but only $2.7 \%$ in neonate weighing over $2,500 \mathrm{~g}(p<0.001)$. Klebsiella bacteremia was more frequent in males than females (32 vs. $19, p=0.04)$. Of 51,22 cases (41\%) of bacteremia were not seriously unwell at the time of blood culture collection. The most common presentation among these cases was hyperbilirubinemia $(15 / 21 ; 71 \%)$. Indeed, hyperbilirubinemia was the single most common presenting feature of Klebsiella bacteremia, occurring in 44 of 51 cases; $57 \%$ of all cases managed by phototherapy and other $43 \%$ by exchange transfusion (-Fig. 1).

Blood cultures collected before the 3rd day of life were positive in 10 of 51 cases (19\%); 25 cases presented between the 3rd and 5th days of life, whereas 16 cases presented at between 5 and 7 days of age (-Fig. 2). As well as hyperbilirubinemia, most patients (68\%) had an elevated CRP concentration; thrombocytopenia (62\%), and leukopenia (20\%) were the other common abnormal laboratory investigations. Near half of patients had all three of the most common abnormal laboratory tests (hyperbilirubinemia, elevated CRP concentration, and thrombocytopenia). Menin-

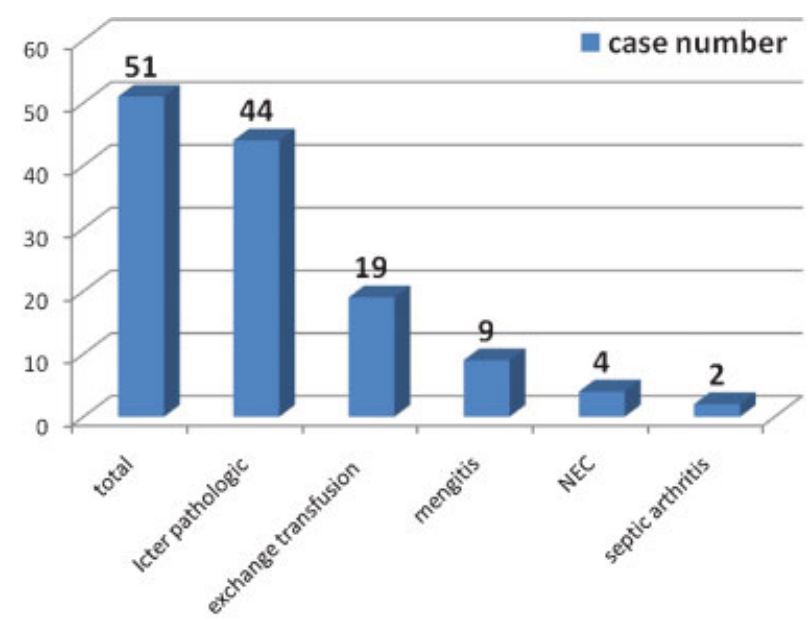

Fig. 1 Complications and presentations of Klebsiella pneumoniae bacteremia in neonates (NEC; necrotizing enterocolitis).

gitis was the most common serious complication, occurring in nine cases (18\%); other complications occurred in seven cases (NEC four cases, septic arthritis two cases, and hydrocephaly one case).

Overall, 13 cases died, giving an overall mortality rate of $25 \%$. Mortality was higher in females and in the lower birth weight neonates; the mortality rate in VLBW neonates $(<1,500 \mathrm{~g})$ was $80 \%$, compared with $23 \%$ in neonates weighing 1,500 to $2,500 \mathrm{~g}$, and $0 \%$ in neonates weighing over 2,500 g group $(p<0.001)$ ( - Fig. 3 ).

\section{Discussion}

Newborns, especially those born preterm, are prone to bacteremia. ${ }^{2}$ Infections may be acquired around the time of birth (early onset), or be acquired after admission to hospital (late onset). ${ }^{3-5}$ Gram-negative bacteremia is a well-recognized cause of nosocomial infection in $\mathrm{NICU}^{14}$; Klebsiella spp. are one of the most common gram-negative bacteria isolated from neonates, causing colonization as well as invasive infections. $^{7,8,15}$ Unsurprisingly, Klebsiella spp. have been

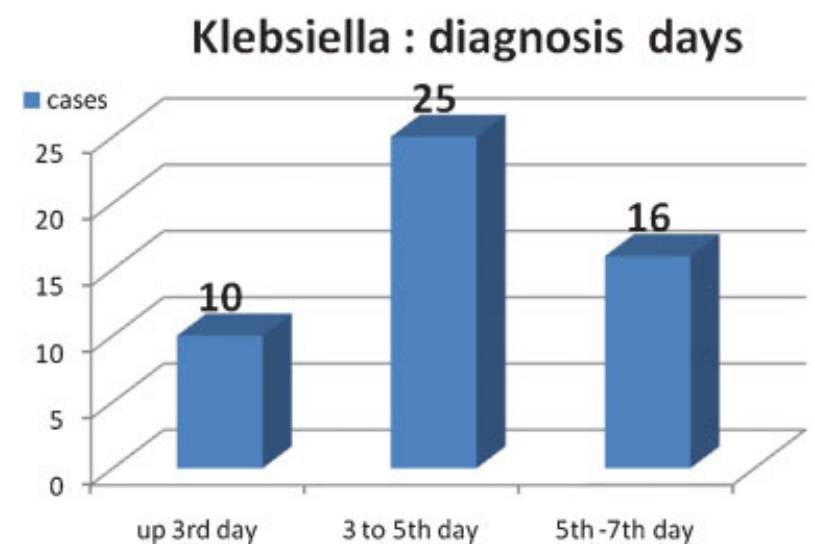

Fig. 2 Time of diagnosis of Klebsiella pneumoniae bacteremia after birth. 


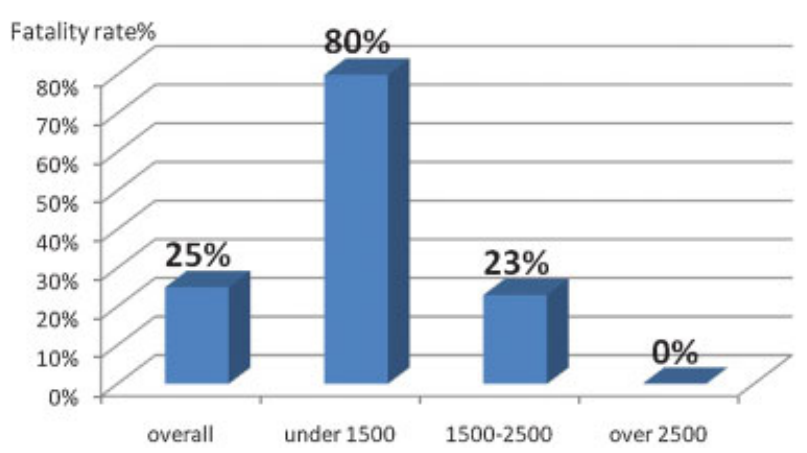

Fig. 3 Mortality rate following Klebsiella pneumoniae bacteremia according to birth weight.

reported to be as the most common cause of neonatal bacteremia in some studies. ${ }^{16}$ It may be due to a strong correlation between colonization of neonates at sites such as the respiratory and gastrointestinal tracts and subsequent development of infection. ${ }^{17}$ The mortality rate in our study (25\%) was lower than in reports of gram-negative bacteremia (Escherichia coli or Klebsiella spp.) in developing countries where mortality rates of around $40 \%$ have been reported. ${ }^{18,19}$ Male gender and prolonged hospital stay have been reported as risk factors for Klebsiella infection, ${ }^{2,20}$ and indeed we found that bacteremia were more common in males. We found that during the first week of life, the risk of Klebsiella bacteremia increased by $15 \%$ per day of hospital stay.

We found that thrombocytopenia was common in patients with Klebsiella bacteremia; other investigators have reported this phenomenon. ${ }^{20}$ In our study, we only included cases presenting in the first week of life, but previous studies have shown that at least $50 \%$ of all cases occur within that time period. ${ }^{8}$ Our finding of a strong correlation between bacteremia and low birth weight reflects the results of previous studies. $^{21}$

In our study, 51 of 805 ( $6 \%$ of all cases) neonates needed to advanced supportive care get infected by Klebsiella. Although late-onset (nosocomially acquired) infection is defined as infection which occurs at least 3 days after admission, ${ }^{22}$ the time period between acquisition of gram-negative bacteria and onset of sepsis can be as short as 1 day in neonates. ${ }^{17}$ Given that all of our infants had a negative blood culture on admission, it is likely that most of the 10 cases of bacteremia that presented within 3 days of birth would have been acquired in the NICU.

However, our overall mortality rate of $25 \%$ masks the birth weight-related differences in mortality; in neonates, weighing less than $1,500 \mathrm{~g}$ the mortality rate was $80 \%$, compared with $0 \%$ in those weighing over $2,500 \mathrm{~g}$. Our findings show the clinical presentation of neonatal Klebsiella bacteremia can be subtle mirrors, the findings of others who have reported that a rising CRP concentration and/or hyperbilirubinemia are common presenting features of neonatal Klebsiella bacteremia. ${ }^{23,24}$ Interestingly, the same studies reported mortality rates of $21 \%$, which is similar to the mortality rate in our study. ${ }^{23,24}$

\section{Conclusion}

K. pneumoniae bacteremia during the first week of life affected $6 \%$ of neonates admitted to our NICU. The mortality rate is high in the VLBW group. It is important to remember that despite the potential lethality of this condition, neonatal Klebsiella bacteremia may present with only nonspecific findings.

\section{Ethical Aspects}

This study was a retrospective review of routine clinical practices and, as such, neither ethics committee approval nor consent was required.

Conflict of Interest

None.

\section{Acknowledgments}

The author thanks Dr. Ali Hafizi and Dr. Fateme Ghotbi for their valuable collaboration in preparing this article.

\section{References}

1 Arbo MD, Snydman DR. Influence of blood culture results on antibiotic choice in the treatment of bacteremia. Arch Intern Med 1994;154(23):2641-2645

2 Moore DL. Nosocomial infections in newborn nurseries and neonatal intensive care units. In: Mayhall CG, ed. Hospital Epidemiology and infection control, 2nd ed. Philadelphia: Lippincott Williams \& Wilkins; 1999:665-693

3 Peter G, Cashore WJ. Infections acquired in the nursery: epidemiology and control. In: Remington JS, Klein JO (Eds.) Infectious diseases of the fetus and newborn infant. 4th ed. Philadelphia: $W$. B. Saunders Co; 1995:1264-1286

4 Stoll BJ, Gordon T, Korones SB, et al. Early-onset sepsis in very low birth weight neonates: a report from the National Institute of Child Health and Human Development Neonatal Research Network. J Pediatr 1996;129(1):72-80

5 Stoll BJ, Hansen N, Fanaroff AA, et al. Late-onset sepsis in very low birth weight neonates: the experience of the NICHD Neonatal Research Network. Pediatrics 2002;110(2 Pt 1):285-291

6 Kim YK, Pai H, Lee HJ, et al. Bloodstream infections by extendedspectrum beta-lactamase-producing Escherichia coli and Klebsiella pneumoniae in children: epidemiology and clinical outcome. Antimicrob Agents Chemother 2002;46(5):1481-1491

7 Hart CA. Klebsiellae and neonates. J Hosp Infect 1993;23(2): 83-86

8 Greenberg D, Shinwell ES, Yagupsky P, et al. A prospective study of neonatal sepsis and meningitis in southern Israel. Pediatr Infect Dis J 1997;16(8):768-773

9 Gladstone IM, Ehrenkranz RA, Edberg SC, Baltimore RS. A ten-year review of neonatal sepsis and comparison with the previous fiftyyear experience. Pediatr Infect Dis J 1990;9(11):819-825

10 Tessin I, Trollfors B, Thiringer K. Incidence and etiology of neonatal septicaemia and meningitis in western Sweden 1975-1986. Acta Paediatr Scand 1990;79(11):1023-1030

11 Vesikari T, Isolauri E, Tuppurainen N, et al. Neonatal septicaemia in Finland 1981-85. Predominance of group B streptococcal infections with very early onset. Acta Paediatr Scand 1989;78(1):44-50 
12 Tullus K, Olsson-Liljequist B, Lundström G, Burman LG. Antibiotic susceptibility of 629 bacterial blood and CSF isolates from Swedish infants and the therapeutic implications. Acta Paediatr Scand 1991;80(2):205-212

13 Cordero L, Sananes M, Ayers LW. Bloodstream infections in a neonatal intensive-care unit: 12 years' experience with an antibiotic control program. Infect Control Hosp Epidemiol 1999;20(4): 242-246

14 Royle J, Halasz S, Eagles G, et al. Outbreak of extended spectrum beta lactamase producing Klebsiella pneumoniae in a neonatal unit. Arch Dis Child Fetal Neonatal Ed 1999;80(1): F64-F68

15 Kühn I, Ayling-Smith B, Tullus K, Burman LG. The use of colonization rate and epidemic index as tools to illustrate the epidemiology of faecal Enterobacteriaceae strains in Swedish neonatal wards. J Hosp Infect 1993;23(4):287-297

16 Misallati A, el-Bargathy S, Shembesh N. Blood-culture-proven neonatal septicaemia: a review of 36 cases. East Mediterr Health J 2000;6(2-3):483-486

17 Hill HR, Hunt CE, Matsen JM. Nosocomial colonization with Klebsiella, type 26, in a neonatal intensive-care unit associated with an outbreak of sepsis, meningitis, and necrotizing enterocolitis. J Pediatr 1974;85(3):415-419
18 Malakan E, Momtazmanesh N. Neonatal sepsis due to Klebsiella: frequency, outcome and antibiotic sensitivity. Iran J Public Health 2004;33(2):43-48

19 Ahmed AS, Chowdhury MA, Hoque M, Darmstadt GL. Clinical and bacteriological profile of neonatal septicemia in a tertiary level pediatric hospital in Bangladesh. Indian Pediatr 2002;39(11): 1034-1039

20 Cordero L, Rau R, Taylor D, Ayers LW. Enteric gram-negative bacilli bloodstream infections: 17 years' experience in a neonatal intensive care unit. Am J Infect Control 2004;32(4):189-195

21 Rodríguez Cervilla J, Fraga JM, García Riestra C, Fernández Lorenzo JR, Martínez Soto I. Neonatal sepsis: epidemiologic indicators and relation to birth weight and length of hospitalization time [in Spanish]. An Esp Pediatr 1998;48(4):401-408

22 Craft A, Finer N. Nosocomial coagulase negative staphylococcal (CoNS) catheter-related sepsis in preterm infants: definition, diagnosis, prophylaxis, and prevention. J Perinatol 2001;21(3):186-192

23 Mai JY, Zhu ML, Chen C, He XL, Lin ZL. Clinical characteristics of neonatal Klebsiella pneumoniae sepsis and the antibiotic sensitivity pattern of strains [in Chinese]. Zhongguo Dang Dai Er Ke Za Zhi 2010;12(9):700-703

24 Maisels MJ, Kring E. Risk of sepsis in newborns with severe hyperbilirubinemia. Pediatrics 1992;90(5):741-743 